ISSN 0103-5150

Fisioter. Mov., Curitiba, v. 23, n. 3, p. 483-492, jul./set. 2010

Licenciado sob uma Licença Creative Commons

\title{
Ação da TENS acupuntural em acupontos na dor induzida pela hipotermia local $\left(0-2^{\circ} \mathrm{C}\right)$
}

\author{
Action of acupunctural TENS in acupuncture points on local \\ bypothermia-induced pain $\left(0-2^{\circ} \mathrm{C}\right)$
}

\section{Eduardo José Nepomuceno Montenegro ${ }^{[a]}$, Nathália Bayma de Albuquerque ${ }^{[b]}$, Larissa Melo Rodrigues de Mariz ${ }^{[\mathrm{c}]}$, Rita de Cássia da Silva Costa ${ }^{[\mathrm{d}]}$, Camila Soares Montarroyos ${ }^{[\mathrm{e}]}$, Mauricy Alves da Motta ${ }^{[\mathrm{f}]}$}

[a] Professor Doutor do Departamento de Fisioterapia da Universidade Federal de Pernambuco (UFPE), Recife, PE - Brasil, e-mail: eduardo.montenegro@ufpe.br

${ }^{[b]}$ Discente do curso de Fisioterapia da Universidade Federal de Pernambuco (UFPE), Recife, PE - Brasil, e-mail: nathbayma@hotmail.com

[c] Discente do curso de Fisioterapia da Universidade Federal de Pernambuco (UFPE), Recife, PE - Brasil, e-mail: laramelomariz@hotmail.com

[d] Discente do curso de Fisioterapia da Universidade Federal de Pernambuco (UFPE), Recife, PE - Brasil, e-mail: ritacassiasilvacosta@yahoo.com.br

[e] Discente do curso de Fisioterapia da Universidade Federal de Pernambuco (UFPE), Recife, PE - Brasil, e-mail: milla_montarroyos@hotmail.com

[f] Professor Doutor do Centro de Ciências Biológicas do Departamento de Biofísica e Radiobiologia da Universidade Federal de Pernambuco (UFPE), Recife, PE - Brasil, e-mail: motta@ufpe.br

\section{Resumo}

Introdução: A dor é um fenômeno multidimensional que dificulta o desenvolvimento das atividades cotidianas. A aplicação de estimulação elétrica nervosa transcutânea (transcutaneous electrical nerve stimulation TENS) está entre uma das modalidades mais usadas para o tratamento da dor. Objetivo: O objetivo deste estudo foi verificar se a TENS acupuntural (baixa frequência) influencia a dor aguda produzida pela hipotermia local em indivíduos saudáveis, quando os eletrodos estão localizados nos acupontos TA5 e CS6 e na aplicação placebo. Metodos: Trinta e dois voluntários, de ambos os gêneros, foram alocados, aleatoriamente, nos grupos TENS e placebo. O estudo consistiu em três momentos: hipotermia sem eletroanalgesia, hipotermia com eletroanalgesia ou placebo, e hipotermia pós-eletroanalgesia ou 
placebo, para avaliar o efeito da TENS e do placebo na latência do limiar de dor e na intensidade da dor. Resultados: Os resultados mostraram que a latência do limiar de dor aumenta significativamente quando comparados os momentos antes e depois do uso da TENS, enquanto o grupo placebo não mostrou significância entre seus ciclos de experimentação. A intensidade da dor não sofreu influencia da TENS e do placebo. Conclusão: Nossos resultados sugerem que a TENS acupuntural aplicada sobre a área dos acupontos TA5 e CS6 é eficaz em aumentar a latência do limiar de dor, mas não interfere na intensidade da dor.

Palavras-chave: Estimulação elétrica nervosa transcutânea. Pontos de acupuntura. Hipotermia local induzida. Dor.

\begin{abstract}
Introduction: Pain is a multidimensional phenomenon that hampers the development of daily activities. The application of transcutaneous electrical nerve stimulation (TENS) is among one of the most widely used to treat pain. Objective: The aim of this study was to determine whether acupunctural TENS (low frequency) influences the acute pain produced by local hypothermia in healthy subjects, when the electrodes are located on acupoints TA5 and CS6 and in placebo application. Methods: Thirty-two volunteers, both male and female, were randomly allocated to TENS groups and sham. The study consisted of three phases: hypothermia without TENS analgesia, hypothermia with TENS analgesia or placebo, and hypothermia post-TENS analgesia or placebo, to evaluate the effect of TENS and placebo in the latency of pain threshold and pain intensity. Results: The results show that the latency of pain threshold significantly increased when compared to the moments before and after use of TENS, while the placebo group showed no significant difference between its cycles of experimentation. Pain intensity was not affected by TENS and placebo. Conclusion: Our results suggest that TENS acupunctural applied over the area of acupoints TA5 and CS6 is effective in increasing the latency of pain threshold, but does not interfere in pain intensity.
\end{abstract}

Keywords: Transcutaneous electrical nerve stimulation. Acupuncture points. Local induced hypothermia. Pain.

\title{
Introdução
}

A dor é um fenômeno multidimensional com componentes sensitivos, emocionais e cognitivos (1) e é descrita, segundo a Associação Internacional para o Estudo da Dor, como uma experiência desagradável, emocional e sensorial, ligada ou não a um dano orgânico ou descrito pelo paciente em tais termos (2). Sua intensidade e frequência geram desconfortos físicos e psicológicos que dificultam o desenvolvimento das atividades cotidianas (1).

Modalidades físicas, tais como estimulação elétrica e térmica, têm sido utilizadas há anos no tratamento da dor. A aplicação de estimulação elétrica nervosa transcutânea (transcutaneous electrical nerve stimulation TENS) está entre uma das modalidades mais comumente usadas para este tratamento (3). A TENS é definida pela Associação de Terapia Física Americana como aplicação terapêutica não medicamentosa de estímulos elétricos na superfície da pele para manejo sintomático de dor aguda e dor crônica de origem benigna (4-6). É um método não invasivo, de baixo custo, seguro e de fácil uso (7) e apresenta poucos efeitos colaterais ou interações medicamentosas (8).

Diferentes teorias foram propostas para o mecanismo de ação da TENS, porém a mais aceita é a Teoria do Controle da Comporta da Dor, de Melzack e Wall (9). De acordo com essa teoria, as informações nociceptivas sofrem concorrência com fibras nervosas que carreiam os estímulos elétricos artificiais para os centros superiores, modulando estas informações álgicas em áreas segmentares e suprassegmentares do sistema nervoso central (8). 
A TENS acupuntural é caracterizada por trabalhar com baixa frequência e alta intensidade, produzindo com isto um abalo muscular $(\leq 10 \mathrm{~Hz})(8,10)$. Esse tipo de TENS tenta mimetizar o efeito mecânico promovido pela introdução da agulha nos pontos acupunturais, por meio de um estímulo elétrico; assim, o indivíduo experimenta uma sensação de parestesia adicionada a abalos musculares, ocorrendo estimulação de fibras tanto mecanorreceptoras como nociceptivas dos grupos III (A $\delta$ ) e IV (C), além de pequenas fibras motoras $(11,12)$.

Este modo de TENS foi desenvolvido para a aplicação da corrente elétrica em pontos acupunturais em sujeitos que apresentam aversão à introdução de agulhas (13).

Métodos alternativos à inserção das agulhas vêm despertando interesses, sendo estudados os estímulos efetuados pela radiação laser, campos magnéticos contínuos (imãs) e campos eletromagnéticos de radiofrequência (14-17). Acreditamos que os estímulos produzidos por correntes elétricas se aproximem mais da natureza bioelétrica dos acupontos, podendo apresentar uma probabilidade maior de respostas por parte destes.

Pelo exposto, temos como proposta neste estudo verificar se a TENS Acupuntural (baixa frequência) influencia o processo álgico agudo produzido pela hipotermia local (frio) em indivíduos hígidos, quando os eletrodos estão localizados nos acupontos TA5 e CS6, e com aplicação placebo, analisando a latência do limiar de dor (em segundos) e a intensidade da dor pela Escala Visual Analógica (EVA).

\section{Metodologia}

\section{Desenho experimental}

O estudo é tipo transversal, unicego e aleatorizado. Ocorreu uma divisão em dois grupos. Cada grupo recebeu 16 voluntários. O primeiro grupo foi estimulado nos pontos TA5 e CS6. O segundo grupo foi estimulado com o placebo.

\section{Sujeitos}

Foram recrutados aleatoriamente 32 voluntários, de ambos os gêneros, com idade entre 18 e 30 anos, que não apresentavam nenhum estado patológico na região submetida à hipotermia, não estavam fazendo uso de medicamentos alopáticos e/ou homeopáticos de qualquer tipo, não sofriam de processo alérgico ou fobia à baixa temperatura e/ou ao gel de acoplamento eletrodo-pele, e no caso de serem do gênero feminino, não estavam em períodos de tensão pré-menstrual e de fluxo catamenial. Os voluntários não tiveram conhecimento prévio do que é a TENS e qual o objetivo do experimento no qual estavam engajados, só obtendo estas respostas no fim de todos os ciclos experimentais. Este trabalho foi aprovado pelo Comitê de Ética em Pesquisa em Seres Humanos do CCS da Universidade Federal de Pernambuco (UFPE), sob o n. 142/07 com base na resolução 196/96 do Conselho Nacional de Saúde (CNS).

\section{TENS}

Foi aplicada a eletroestimulação com TENS acupuntural $(10 \mathrm{~Hz})$ com forma de onda bifásica assimétrica balanceada, gerada pelo equipamento TENSYS ET $9771 \mathrm{KLD}^{\circledR}$ com dois canais de saída, com duração de pulso de $1 \mathrm{~ms}$ (milisegundo) e intensidade que produzisse um abalo muscular visível na região onde os eletrodos foram posicionados. Este pulso foi administrado por meio de um canal com dois eletrodos de material à base de borracha siliconada impregnada de carbono, com área de $15 \mathrm{~cm}^{2}$ (cada eletrodo), os quais foram acoplados à região selecionada por meio de gel à base de água e fixados com esparadrapo. Um grupo composto de 16 voluntários (nove homens e sete mulheres) foi estimulado com a TENS acupuntural nos pontos TA5 e CS6, localizados a dois cun (1 cun corresponde à largura do dedo polegar do voluntário em 
sua parte mais larga) acima da prega do punho não dominante, na porção posterior e anterior do antebraço, respectivamente. Os demais voluntários (dez homens e seis mulheres) foram engajados no grupo placebo. A intensidade da corrente elétrica utilizada foi suficiente para provocar abalos musculares nos voluntários.

\section{Hipotermia local induzida}

Inicialmente, o voluntário imergiu sua mão não dominante em um recipiente com água morna $\left(37^{\circ} \mathrm{C}\right)$ durante cinco minutos. Após esse tempo, a mão foi retirada do recipiente e imersa em outro com água gelada a $0-2^{\circ} \mathrm{C}$ (o controle da temperatura foi feito por meio do termômetro de mercúrio Incoterm L-212/04 ${ }^{\circledR}$ ). A latência de tempo entre o momento em que o indivíduo colocou a mão no recipiente com água gelada e o momento em que referiu à sensação "dor" representou a medida da latência do limiar de dor (registrado pelo cronômetro digital Cronobio SW2018 ${ }^{\circledR}$ ). Quando o voluntário referiu a sensação "dor", foi solicitado que ele permanecesse com a mão na água gelada por mais 30 segundos (alguns voluntários não concluíram este tempo). Após a retirada do membro do recipiente com água gelada foi solicitado ao voluntário que descrevesse a sensação dolorosa por meio de Escala Visual Analógica (valores de 0 a 10, em que 0 não representa dor alguma e 10 representa a dor máxima suportável), caracterizando assim os valores da intensidade da dor. Após um intervalo de descanso de cinco minutos, esse procedimento - água morna/ água gelada - foi repetido novamente, completando, com isso, o ciclo de pré-tratamento (composto por dois subciclos de estimulação), com duração de 20 minutos [metodologia citada por Johnson e Tabasam (18)]. Antes desse procedimento, os indivíduos foram testados para constatar ou não possíveis alergias à baixa temperatura ou distúrbios (fobia) comportamentais ao estímulo térmico. Nenhum indivíduo apresentou processo alérgico aos estímulos dados.

\section{Eletroanalgesia}

Em prosseguimento ao ciclo experimental, o voluntário colocou novamente a mão dentro do recipiente com água morna durante cinco minutos, agora com a TENS ligada nos acupontos TA5 e CS6. Passados os cinco minutos, o voluntário retirou a mão da água morna e colocou no recipiente com a água gelada a $0-2^{\circ} \mathrm{C}$. Foram medidas a latência do limiar de dor (cronômetro) e a intensidade da dor, por meio da EVA, como já referido anteriormente no ciclo de pré-tratamento. Esse procedimento foi repetido também em dois subciclos, com cada um perdurando por aproximadamente dez minutos, completando o ciclo de tratamento. Com isso, no fim dos dois subciclos com a TENS ligada, cada voluntário recebeu 20 minutos de eletroestimulação. Ao término deste ciclo de tratamento, o voluntário foi submetido novamente a mais dois subciclos - ciclo de póstratamento -, seguindo o mesmo método já citado, agora com a TENS desligada e para avaliação do efeito da TENS pós-tratamento. Dessa forma, todo o processo experimental durou 60 minutos. Cada voluntário passou por esses ciclos de pré-tratamento, tratamento e pós-tratamento.

\section{Placebo}

No estado experimental placebo, um grupo de 16 voluntários (dez homens e seis mulheres) foi submetido ao procedimento de hipotermia e de eletroanalgesia citados anteriormente, porém os voluntários não receberam estímulo elétrico, mas foram induzidos a pensar que estavam sendo submetidos a um tratamento de eletroestimulação de microcorrente, no qual não sentiriam nenhuma forma de sensação de formigamento, abalo muscular ou algo parecido. Foi explicado ao grupo que a microcorrente trabalha com intensidades muito baixas, com grande poder de analgesia e não sendo perceptível (sem sensação vibratória). Os voluntários foram conectados a um primeiro canal com os dois eletrodos posicionados na articulação do ombro não dominante, mas foi ligado no aparelho (Tensys ET $9771 \mathrm{KLD}^{\circledR}$ ) o segundo canal. Com isso, o indivíduo percebeu que o

equipamento estava ligado (luzes acesas), sendo levado a crer que as microcorrentes estavam atuando em seu 
corpo. Os locais da articulação do ombro, onde foram colocados os eletrodos, não possuem nenhum ponto acupuntural ou ponto reflexo que atue como acupontos. O grupo placebo também foi submetido aos três ciclos de experimentação, com duração total de 60 minutos.

\section{Análise estatística}

Os dados da latência do limiar de dor e da intensidade da dor estão apresentados como média aritmética \pm desvio padrão. Foi utilizado o Teste de ANOVA de dois critérios para comparação entre os ciclos de cada grupo (TENS e placebo), em relação à latência do limiar de dor, seguido, quando necessário, do Teste post hoc de Student-Newman-Keuls. Para a análise dos dados dos ciclos referentes à intensidade da dor foi efetuado o teste de Kruskal-Wallis. A significância adotada nesse estudo foi de p < 0,05.

\section{Resultados}

Na Tabela 1 estão apresentados os dados da latência do limiar de dor dos voluntários (sujeitos) submetidos à TENS nos acupontos TA5 e CS6. No ciclo de pré-tratamento, a latência do limiar de dor (segundos) foi de 26,71 \pm 9,86; durante o tratamento (TENS ligada), de 30,09 \pm 12,35; e no pós-tratamento (TENS desligada), de 34,28 \pm 13,23. A análise desses resultados demonstrou significância tanto no tratamento - ANOVA; Student-Newman-Keuls - como também entre os indivíduos - Student-Newman-Keuls (os dados dos indivíduos não foram apresentados). Analisando os ciclos pré-tratamento $(26,71 \pm 9,86)$ e durante o tratamento $(30,09 \pm 12,35)$, não foi verificada significância no resultado. No ciclo pré-tratamento e pós-tratamento $(34,28 \pm 13,23)$ foram constatados efeitos significantes, mas que não se repetiu quando comparado o ciclo durante o tratamento com o pós-tratamento.

Tabela 1 - Latência do limiar de dor (em segundos) dos ciclos de experimentação do grupo TENS Acupuntural

\begin{tabular}{ccc}
\hline Ciclos & Média \pm DP (segundos) & Estatística* \\
\hline Pré-tratamento/ & $26,71 \pm 9,86 /$ & $\mathrm{p}>0,05$ \\
Tratamento & $30,09 \pm 12,35$ & \\
Tratamento/ & $30,09 \pm 12,35 /$ & $\mathrm{p}>0,05$ \\
Pós-tratamento & $34,28 \pm 13,23$ & \\
Pré-tratamento/ & $26,71 \pm 9,86 /$ & $\mathrm{p}<0,05^{*}$ \\
Pós-tratamento & $34,28 \pm 13,23$ & \\
\hline
\end{tabular}

*ANOVA - post hoc Student-Newman-Keuls (análise entre os sujeitos apresentou significância, dados não apresentados).

$\mathrm{Na}$ Tabela 2 estão apresentados os dados referentes à intensidade da dor dos voluntários submetidos à TENS nos acupontos TA5 e CS6. No ciclo de pré-tratamento, a intensidade da dor (EVA) foi de 6,79 $\pm 1,5$; durante o tratamento, de 8,12 $\pm 1,46$; e no pós-tratamento, de 7,75 $\pm 1,83$. Após análise dos dados - teste de Kruskal-Wallis - foi constatado que não ocorreu efeito significante na intensidade da dor com o uso da TENS acupuntural. 
Tabela 2 - Intensidade da dor (EVA) dos ciclos de experimentação do grupo TENS Acupuntural

\begin{tabular}{ccc}
\hline Ciclos & Média \pm DP (EVA) & Estatística* \\
\hline Pré-tratamento/ & $6,79 \pm 1,5 /$ & $\mathrm{p}>0,05$ \\
Tratamento & $8,12 \pm 1,46$ & \\
Tratamento/ & $8,12 \pm 1,46 /$ & $\mathrm{p}>0,05$ \\
Pós-tratamento & $7,75 \pm 1,83$ & \\
Pré-tratamento/ & $6,79 \pm 1,5 /$ & $\mathrm{p}>0,05$ \\
Pós-tratamento & $7,75 \pm 1,83$ & \\
\hline
\end{tabular}

*Teste de Kruskal-Wallis.

$\mathrm{Na}$ Tabela 3 estão apresentados os dados referentes à latência do limiar de dor dos voluntários submetidos ao grupo placebo. No ciclo pré-tratamento, a latência do limiar de dor foi de 34,41 \pm 11,80; durante a indução do placebo, de 44,99 \pm 24,78; e no pós-tratamento, de 43,38 $\pm 32,28$. Após análise dos dados, foi verificado que não ocorreu diferença significativa entre os ciclos de experimentação (pré, durante e pós), mas ocorreu diferença significativa - ANOVA; Student-Newman-Keuls - entre os voluntários (dados não apresentados).

Tabela 3 - Latência do limiar de dor (em segundos) dos ciclos de experimentação do grupo placebo

\begin{tabular}{ccc}
\hline Ciclos & Média \pm DP (segundos) & Estatística* \\
\hline Pré-tratamento/ & $34,41 \pm 11,80 /$ & $\mathrm{p}>0,05$ \\
Tratamento & $44,99 \pm 24,78$ & \\
Tratamento/ & $44,99 \pm 24,78 /$ & $\mathrm{p}>0,05$ \\
Pós-tratamento & $43,38 \pm 32,28$ & \\
Pré-tratamento/ & $34,41 \pm 11,80 /$ & $\mathrm{p}>0,05$ \\
Pós-tratamento & $43,38 \pm 32,28$ & \\
\hline
\end{tabular}

*ANOVA - post hoc Student-Newman-Keuls (análise entre os sujeitos apresentou significância, dados não apresentados).

$\mathrm{Na}$ Tabela 4 estão apresentados os dados referentes à intensidade da dor dos voluntários submetidos ao grupo placebo. No ciclo pré-tratamento o valor foi de 8,12 \pm 0,92; durante o placebo, de 7,96 \pm 1 ,4; e no pós-tratamento, de 7,79 $\pm 1,54$. Após análise dos dados - teste de Kruskal-Wallis -, foi verificado que não ocorreu significância entre os ciclos de experimentação. 
Tabela 4 - Intensidade da dor (EVA) dos ciclos de experimentação do grupo placebo

\begin{tabular}{ccc}
\hline Ciclos & Média \pm DP $($ EVA) & Estatística* \\
\hline Pré-tratamento/ & $8,12 \pm 0,92 /$ & $\mathrm{p}>0,05$ \\
Tratamento & $7,96 \pm 1,4$ & \\
Tratamento/ & $7,96 \pm 1,4 /$ & $\mathrm{p}>0,05$ \\
Pós-tratamento & $7,79 \pm 1,54$ & \\
Pré-tratamento/ & $8,12 \pm 0,92 /$ & $\mathrm{p}>0,05$ \\
Pós-tratamento & $7,79 \pm 1,54$ & \\
\hline
\end{tabular}

*Teste de Kruskal-Wallis.

\section{Discussão}

A latência do limiar de dor e a intensidade da dor são fenômenos neurofisiológicos distintos, a primeira no âmbito sensorial e a segunda no âmbito afetivo (psicológico) (3). Com isso, possuem representações distintas durante a interpretação desta sensação (dor) diante do estímulo álgico. Verificamos que no grupo da TENS, nos acupontos TA5 e CS6, os voluntários apresentaram um aumento na latência do limiar de dor no ciclo de pós-tratamento, em relação ao pré-tratamento, mas não em relação ao tratamento (Tabela 1). Os pontos TA5 e CS6 são estimulados com agulhas, sementes ou com a eletroacupuntura, em pacientes que apresentam, entre outras causas, má circulação periférica nas mãos, alterações na circulação sistêmica e falha na desobstrução do sangue (19), pois a acupuntura parece reduzir o tônus neurovegetativo simpático, resultando em melhora da perfusão periférica local e geral em seres humanos (20).

A hipotermia local induzida utilizada neste estudo promove a sensação álgica por meio da vasoconstricção promovida no local $(18,21)$, em que o estímulo da TENS acupuntural nesses acupontos desencadeou um aumento na latência do limiar sensorial após a aplicação. A TENS acupuntural atua na liberação de opioides endógenos, ativação da via serotoninérgica, noradrenérgica e adrenérgica ( $\beta$-endorfina, serotonina, noradrenalina, adrenalina, etc.) (22), usando o mesmo mecanismo que a eletroacupuntura, que por sua vez aumenta os níveis de $\beta$-endorfina e encefalina no LCR (23). Um dos opioides mais potentes é a dinorfina, que atua nos receptores opioides $\mu$. Receptores muscarínicos (subtipos $\mathrm{M}_{1}$ e $\mathrm{M}_{3}$ ) também são ativados pela TENS acupuntural $(24,25)$; com isto, promovem sensações analgésicas mais prolongadas, podendo se estender até 12 horas após a aplicação (26).

No nosso estudo a avaliação foi feita antes, durante e após aplicação, perfazendo um período relativamente curto de 20 minutos de aplicação, com 20 minutos de avaliação pós-aplicação. Mesmo com o tempo pequeno de mensuração na pós-aplicação, e como a TENS não estava atuando neste momento, podemos descartar a ação da TENS na região segmentar, sugerindo que estes mecanismos citados (suprassegmentar) podem ter sido deflagrados para promover o aumento na latência do limiar de dor quando os eletrodos estavam localizados nos pontos acupunturais anteriormente citados. Não temos evidências de que a TENS tenha atuado exclusivamente nos pontos acupunturais, pois os eletrodos possuem área que abrange o nervo mediano, ulnar e radial, mas, pelo posicionamento dos eletrodos em cima dos acupontos, sabemos que a corrente elétrica passou por eles. Isto corrobora um estudo de Nayak et al (27), que conseguiram um aumento no estado de sedação de pacientes de UTI, sem qualquer efeito colateral, com eletroestimulação superficial de acupontos.

Entre os voluntários, foi detectada diferença significante nos seus dados, evidenciando que os sujeitos apresentam respostas diferentes ao estímulo da TENS quando aplicada nesses acupontos, provavelmente causadas por características individuais, e, desta forma, sua explicação foge ao objetivo deste trabalho. 
A análise da intensidade da dor por meio da EVA nos voluntários submetidos à TENS (Tabela 2) e ao placebo (Tabela 4) demonstrou que não houve alterações no âmbito psicológico na sensação da dor dentro de cada grupo, ou seja, a discriminação afetiva não alterou durante os ciclos experimentais, tanto com a TENS como com o placebo. As vias de discriminação desses dois estados neurofisiológicos (sensorial e psicológico) são distintas, como afirmou Solomon et al (3). Nosso resultado corrobora essa evidência, pois os resultados entre a latência do limiar de dor e a intensidade da dor foram distintos.

A latência do limiar de dor no grupo placebo (Tabela 3) não apresentou significância entre os ciclos de experimentação, mas apresentou entre os indivíduos, como pode ser constatado pela grande variação do desvio padrão e dos testes estatísticos. Alguns voluntários não apresentaram resposta ao placebo, mas outros foram sugestionados a acreditar que aquele procedimento com a TENS ("microcorrente") iria reduzir substancialmente a dor promovida pela hipotermia.

Pesquisadores (28) demonstraram que o efeito placebo pode estar ligado à origem etnocultural, no qual foi constatado que grupos asiáticos são mais susceptíveis a estímulos de indução placebo do que grupos anglo-saxônicos. Nós sugerimos também que até dentro da mesma origem etnocultural, determinados indivíduos apresentam uma sugestionabilidade maior. Sabe-se que o estímulo placebo ativa no cérebro os mesmos circuitos ativados por estímulos analgésicos (químicos, mecânicos e elétricos). Os estímulos placebo e analgésicos produzem um aumento no fluxo sanguíneo em áreas ricas em receptores de opioides que mantêm contato com outras regiões do cérebro, incluindo o córtex órbito-frontal, área que também processa as emoções. Assim, pessoas que apresentam respostas mais intensas ao placebo possivelmente possuem esse sistema de receptores mais eficientes, com maior concentração de elementos (29). Campbell (30) relata que há evidência que placebo e hipnose podem produzir mudanças cerebrais complexas em áreas conectadas com transmissão e percepção da dor e que efeitos similares ocorrem em resposta à acupuntura.

Apesar de apresentar resultado estatístico entre os indivíduos submetidos ao placebo, quando efetuada a média aritmética e comparados os momentos antes, durante e após a indução, verifica-se que não ocorre significância dentro do grupo nestes momentos analisados. Provavelmente para se obter um melhor quadro de analise sobre o efeito placebo, seja necessário um número grande de sujeitos engajados nos grupos experimentais.

\section{Considerações finais}

Apesar de a TENS acupuntural aumentar a latência do limiar de dor dos sujeitos, não podemos afirmar que este resultado foi decorrente apenas da estimulação dos acupontos TA5 e CS6. Dessa forma, sugerimos estudos posteriores com a colocação de eletrodos pontuais (com área menor) sobre os acupontos usados no presente estudo para observar como a estimulação elétrica pontual irá atuar.

Nossos resultados sugerem que a TENS acupuntural aplicada na área que envolva os acupontos TA5 e CS6 é eficaz em aumentar a latência do limiar de dor em indivíduos saudáveis, sugerindo que mecanismos centrais para o alívio da dor foram ativados. Recomendamos essa aplicação em processos patológicos que tenham como quadro secundário a dor provocada por vasoconstricção na mão.

\section{Referências}

1. Frutuoso JT, Cruz RM. Relato verbal na avaliação psicológica da dor. Aval Psicol. 2004;3:107-14.

2. Horn, A. Reflexão sobre a dor. Anais do II Encontro Mundial dos Estados Gerais da Psicanálise; 2003; Rio de Janeiro, Brasil. Rio de Janeiro; 2003. Disponível em: <http://www.estadosgerais.org/mundial_rj/download/5c_ Horn_106141003_port.pdf>. Acesso em: 25 jan. 2009.

3. Solomon J, Shebshacvich V, Adler R. The effects of TENS, heat and cold on the pain thresholds induced by mechanical pressure in healthy volunteers. Neuromodulation. 2003;2:102-7. 
4. Woolf C, Thompson J. Segmental afferent fiber-induced analgesia: transcutaneous eletrical nerve stimulation (TENS) and vibration. In: Wall P, Melzack R. Textbook of pain. New York: Churchill Livingstone; 1994. p. 884-96.

5. Walsh D. Non-analgesic effects of TENS. In: Walsh, D. TENS clinical applications and related theory. New York: Churchill Livingstone; 1997. p. 157-62.

6. Kalra A, Urban MO, Sluka KA. Blockade of opioid receptors in rostral ventral medulla prevents antihiperalgesia produced by transcutaneous electrical nerve stimulation (TENS). J Pharmacol Exp Ther. 2001;298:257-63.

7. American Physical Therapy Association - APTA. Electrotherapy Standards Committee: electrotherapeutic terminology in physical therapy (report). Alexandria, VA: APTA; 2001.

8. Radhakrishnan R, Sluka KA. Deep tissue afferents, but not cutaneous afferents, mediate transcutaneous electrical nerve stimulation-induced antihyperalgesia. J Pain. 2005;6:673-80.

9. Sluka KA, Vance CGT, Lisi TL. High-frequency, but not low-frequency, transcutaneous electrical nerve stimulation reduces aspartate and glutamate release in the spinal cord dorsal horn. J Neurochem. 2005a;95:1794-801.

10. Robinson AJ, Snyder-Mackler L. Clinical electrophysiology: electrotherapy and electrophysiological testing. 2nd ed. Baltimore, MD: Williams and Wilkins; 1995.

11. WalshDM. Transcutaneous electrical nerve stimulation and acupuncture points. Complement Therap Med. 1996;4:133-37.

12. Tomasso M, Fiore P, Camporeale A, Guido M, Libro G, Losito L, et al. High and low frequency transcutaneous electrical nerve stimulation inhibits nociceptive responses induced by CO2LASER stimulation in humans. Neurosci Lett. 2003;342(1-2):17-20.

13. Amestoy RDF. Eletroterapia e eletroacupuntura: princípios básicos... e algo mais. 2a ed. Florianópolis: Absoluta; 2005.

14. Ebneshahidi NS, Heshmatipour M, Moghaddami A, Araghi PE. The effects of laser acupuncture on chronic tension headache: a randomized controlled trial. Acupunct Med. 2005;23(1):13-8.

15. Hübscher M, Vogt L, Banzer W. Wavelet analysis of laser-induced blood flow changes. Medical Acupuncture. 2007;19(1):13-6.

16. ZeredoJL, Sasaki KM, Toda K. High-intensity laser for acupuncture-like stimulation. Lasers Med Sci. 2007;22(1):37-41.

17. Colbert AP, Cleaver J, Brown AB, Harling N, Hwang Y, Shiffke HC. Magents applied to acupuncture points as therapy- a literature rewiew. Acupunctur Med. 2008;26(3):60-70.

18. Johnson MI, Tabasam G. An Investigation into the analgesic effects of different frequencies of the amplitudemodulated wave of interferential current therapy on cold-induced pain in normal subjects. Arch Phys Med Rehabil. 2003;84(9):1387-94.

19. Cross JR. Acupressure: clinical applications in musculo-skeletal conditions. Reed Educational \& Professional Publishing; 2000. p. 189.

20. Schikora D. Laserneedle acupuncture: a critical rewiew and recent results. Med Acupunct. 2008;20(1):37-42.

21. Tulgar M, Tulgar O, Herken H. Psychophysical responses to experimentally induced heat and cold pain before, during, and after Transcutaneous Electrical Nerve Stimulation. Neuromodulation. 2003;6:229-36.

22. Sluka KA, King EW, Audette K. Transcutaneous electrical nerve stimulation activates peripherally located alpha2A adrenergic receptors. Pain. 2005;115(3):364-73.

23. Taguchi T, Taguchi R. Effect of varying frequency and duration of electroacupuncture stimulation on carrageenaninduced hyperalgesia. Acupunct Med. 2007;25(3):80-6.

24. Radhakrishnan R, Sluka K. Spinal muscarinic receptors are activated during low or high frequency TENS-induced antihyperalgesia in rats. Neuropharmacology. 2003;45(8):1111-9. 
25. Sluka KA, Lisi TL, Westlund KN. Increased release of serotonin in the spinal cord during low, but not high, frequency transcutaneous electric nerve stimulation in rats with joint inflammation. Arch Phys Med Rehabil. 2006;87:1137-140.

26. Sluka KA, Bailey K, Bogush J. Treatment with either high or low frequency TENS reduces the secondary hyperalgesia observed after injection of kaolin and carrageenan into the knee joint. Pain. 1998;7:97-102.

27. Nayak S, Wenstone R, Jones A. Surface electrostimulation of acupuncture points for sedation of critically ill patients in the intensive care unit: a pilot study. Acupunct Med. 2008;26(1):1-7.

28. Johnson M, Din A. Ethnocultural differences in the analgesic effects of placebo transcutaneous electrical nerve stimulation on cold-induced pain in healthy subjects: a preliminary study. Complement Ther Med. 1997;5:74-9.

29. Holden C. Drugs and placebos look alike in the brain. Science. 2002;295(5557):947.

30. Campbell A. Point specificity of acupuncture in the light of recent clinical and imaging studies. Acupunct Med. 2006;24(3):118-22.

Recebido: 26/02/2009

Received: 02/26/2009

Aprovado: 04/05/2010

Approved: 05/04/2010

Revisado: 25/06/2010

Reviewed: 06/25/2010 